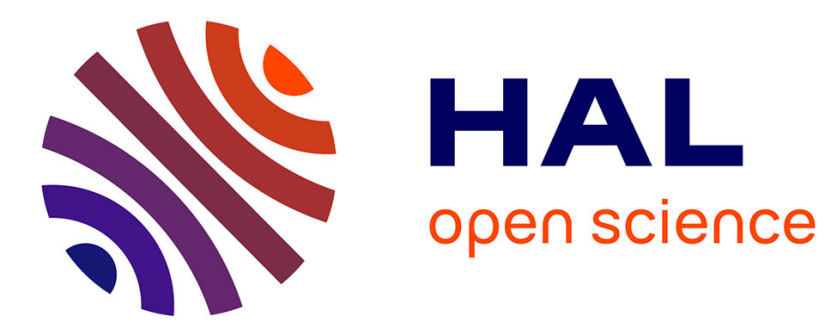

\title{
Bayesian parameter estimation for asymmetric power distributions
}

Alexandre Baussard, Jean-Yves Tourneret

\section{To cite this version:}

Alexandre Baussard, Jean-Yves Tourneret. Bayesian parameter estimation for asymmetric power distributions. 23rd European Signal Processing Conference (EUSIPCO 2015), Aug 2015, Nice, France. pp. 2206-2210. hal-01485027

\section{HAL Id: hal-01485027 \\ https://hal.science/hal-01485027}

Submitted on 8 Mar 2017

HAL is a multi-disciplinary open access archive for the deposit and dissemination of scientific research documents, whether they are published or not. The documents may come from teaching and research institutions in France or abroad, or from public or private research centers.
L'archive ouverte pluridisciplinaire HAL, est destinée au dépôt et à la diffusion de documents scientifiques de niveau recherche, publiés ou non, émanant des établissements d'enseignement et de recherche français ou étrangers, des laboratoires publics ou privés. 


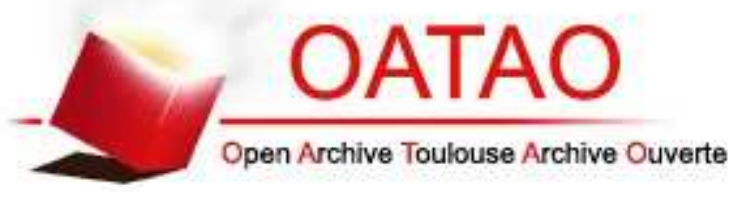

\section{Open Archive TOULOUSE Archive Ouverte (OATAO)}

OATAO is an open access repository that collects the work of Toulouse researchers and makes it freely available over the web where possible.

This is an author-deposited version published in : http://oatao.univ-toulouse.fr/ Eprints ID : 17105

The contribution was presented at EUSIPCO 2015 :

http://www.eusipco2015.org/

To cite this version : Baussard, Alexandre and Tourneret, Jean-Yves Bayesian parameter estimation for asymmetric power distributions. (2015) In: 23rd European Signal Processing Conference (EUSIPCO 2015), 31 August 2015 - 4 September 2015 (Nice, France).

Any correspondence concerning this service should be sent to the repository administrator: staff-oatao@listes-diff.inp-toulouse.fr 


\title{
BAYESIAN PARAMETER ESTIMATION FOR ASYMMETRIC POWER DISTRIBUTIONS
}

\author{
Alexandre BAUSSARD ${ }^{(1,2)}$ and Jean-Yves TOURNERET ${ }^{(2)}$ \\ ${ }^{(1)}$ Lab-STICC (UMR CNRS 6285), ENSTA Bretagne, 2 rue François Verny, 29806 Brest cédex 9, France. \\ ${ }^{(2)}$ IRIT (UMR CNRS 5505), INP-ENSEEIHT, 2 rue Charles Camichel, 31000 Toulouse, France.
}

\begin{abstract}
This paper proposes a hierarchical Bayesian model for estimating the parameters of asymmetric power distributions (APDs). These distributions are defined by shape, scale and asymmetry parameters which make them very flexible for approximating empirical distributions. A hybrid Markov chain Monte Carlo method is then studied to sample the unknown parameters of APDs. The generated samples can be used to compute the Bayesian estimators of the unknown APD parameters. Numerical experiments show the good performance of the proposed estimation method. An application to an image segmentation problem is finally investigated.
\end{abstract}

Index Terms - Asymmetric power distributions, hierarchical Bayesian model, MCMC, Gibbs sampler, Image segmentation.

\section{INTRODUCTION}

Many image processing applications require to define an appropriate probability distribution for the observed data. These applications include image classification, image segmentation, image registration or change detection. A very classical approach for defining a probability density function (pdf) for observed data is to fit the empirical histogram of these data by classical pdfs such as Gaussian, gamma, Laplace or by their generalized versions. In particular, the symmetric generalized Gaussian distribution has received a considerable attention in the literature because it generalizes classical distributions such as the Gaussian or Laplace distributions. However, in some other applications such as econometrics $[1,2]$ or image processing, asymmetry has been observed in the distribution of the data. In particular, image processing applications involving asymmetric distributions include segmentation of magnetic resonance images (MRI) [3] or texture classification using wavelet coefficients [4].

This paper studies asymmetric power distributions (APDs) that have been introduced in $[1,2]$. These distributions are characterized by scale, shape and asymmetry parameters which make them more flexible than the symmetric distributions for approximating empirical histograms. We are particularly interested in developing a Bayesian parameter estimation method for this kind of distributions. More precisely, all the unknown parameters of APDs are assigned prior distributions summarizing the known information about these parameters. A Markov chain Monte Carlo (MCMC) method is then introduced to sample the resulting posterior distribution and to compute the Bayesian estimators of the unknown APD parameters. The performance of the proposed Bayesian estimation method is evaluated via several simulation results. An application to the segmentation of images with APDs is finally investigated.

The paper is organized as follows. The asymmetric power distributions considered in this work are introduced in Section 2. Section 3 presents the Bayesian model suggested for estimating the APD parameters. The Bayesian estimators associated with this model being difficult to compute in closedform, we study in section 4 an MCMC approach that can be used to sample according to the posterior of the unknown APD parameters. The performance of this approach is evaluated via simulated results associated with synthetic data. Section 5 considers an application of the proposed method for image segmentation. Section 6 gives some concluding remarks and future work.

\section{ASYMMETRIC POWER DISTRIBUTIONS (APDS)}

We consider a class of univariate asymmetric power distributions defined by the following pdf

$f_{\mathrm{APD}}(x \mid \boldsymbol{\theta})= \begin{cases}\frac{\delta^{1 / \lambda}}{\gamma^{1 / \lambda} \Gamma(1+1 / \lambda)} \exp \left(-\frac{\delta}{\gamma \alpha^{\lambda}}|x|^{\lambda}\right), & \text { if } x \leq 0 \\ \frac{\delta^{1 / \lambda}}{\gamma^{1 / \lambda} \Gamma(1+1 / \lambda)} \exp \left(-\frac{\delta}{\gamma(1-\alpha)^{\lambda}}|x|^{\lambda}\right), & \text { if } x>0\end{cases}$

where $\boldsymbol{\theta}=(\lambda, \gamma, \alpha)^{T}$ is a vector containing the APD parameters, $\Gamma($.$) is the gamma function and \delta=\frac{2 \alpha^{\lambda}(1-\alpha)^{\lambda}}{\alpha^{\lambda}+(1-\alpha)^{\lambda}}$.

The shape of the APD distribution is adjusted by the parameter $\lambda>0$ controlling the tail decay whereas $\alpha \in(0,1)$ characterizes the degree of asymmetry and $\gamma>0$ is a scale parameter. Some related APD definitions that are equivalent up to an appropriate change of variables can also be found in $[1,2,4]$.

The proposed distribution defined by (1) has two main advantages (for our purpose) with respect to APDs of [1] or [4]: the asymmetric parameter is constrained to belong to a finite length interval $(0,1)$ and the presence of a scale parameter makes it more flexible for practical applications. 


\section{BAYESIAN PARAMETER ESTIMATION}

This section studies a Bayesian method for estimating the parameters $(\alpha, \lambda, \gamma)$ of an APD. The motivation for using this method is that it is generic and can be applied to many problems involving APDs. In particular, the image segmentation problem considered in Section 5 can be handled by a similar method. Conversely, the maximum likelihood method proposed in [1] cannot be easily applied to the image segmentation problem which requires to estimate discrete and continuous parameters. The principle of parameter estimation using Bayesian inference is to define appropriate priors for the unknown parameters (and possibly hyperparameters) and to estimate these parameters using their posterior distribution. The priors considered in this study are summarized below.

\subsection{Prior distributions}

According to the APD pdf given in equation (1), the shape parameter $\lambda$ is defined on $\mathbb{R}^{+}$. However, in practical problems, its range can be reduced to $[0,3][5]$. Thus, one can assign to $\lambda$ the uniform prior

$$
p(\lambda)=\frac{1}{3} \mathbb{I}_{[0,3]}(\lambda)
$$

The scale parameter is assigned a Jeffreys prior

$$
p(\gamma)=\frac{1}{\gamma} \mathbb{I}_{\mathbb{R}^{+}}(\gamma)
$$

This choice of non-informative prior is very classical for scale parameters (see [6] for motivations).

The asymmetry parameter $\alpha$ is constrained in the interval $(0,1)$. When there is no additional information, it is natural to choose the following uniform prior for this parameter

$$
p(\alpha)=\mathbb{I}_{(0,1)}(\alpha) .
$$

\subsection{Posterior distribution}

We assume that the parameters $\alpha, \lambda$ and $\gamma$ are a priori independent. For any sample $\boldsymbol{x}=\left(x_{1}, \ldots, x_{n}\right)^{T} \in \mathbb{R}^{n}$ distributed according to an APD with unknown parameter vector $\boldsymbol{\theta}=(\lambda, \gamma, \alpha)^{T}$, the posterior distribution of $\boldsymbol{\theta}$ can be written

$$
p(\boldsymbol{\theta} \mid \boldsymbol{x}) \propto\left(\prod_{i=1}^{n} p\left(x_{i} \mid \boldsymbol{\theta}\right)\right) p(\lambda) p(\gamma) p(\alpha)
$$

where $\propto$ means "proportional to". This posterior is too complex to derive closed-form expressions of the Bayesian estimators of $\boldsymbol{\theta}$. As a consequence, we propose to use an MCMC method to generate samples asymptotically distributed according to (5) and to use the generated samples to build estimators of the unknown parameters. It is the objective of the next section.

\section{HYBRID GIBBS SAMPLER}

The principle of MCMC methods is to construct a Markov chain whose equilibrium distribution is the target posterior distribution. In order to do that, the basic Gibbs sampler generates samples according to the conditional distributions of the target distribution. When the conditional distribution of a subvector of the unknown parameter vector cannot be sampled easily, we can generate this subvector according to an appropriate proposal and accept or reject this generated vector using the Metropolis acceptance ratio. When Metropolis moves are used inside a Gibbs sampler, the resulting MCMC method is referred to as Metropolis-within-Gibbs or hybrid Gibbs sampler. This strategy can be used to generate samples distributed according to (5) by sampling according to the conditional distributions $p(\lambda \mid \boldsymbol{x}, \gamma, \alpha), p(\gamma \mid \boldsymbol{x}, \lambda, \alpha)$ and $p(\alpha \mid \boldsymbol{x}, \lambda, \gamma)$, which are detailed below.

\subsection{Sampling the shape parameter $\lambda$}

The conditional distribution of the shape parameter $\lambda$ satisfies the following relation

$$
p(\lambda \mid \boldsymbol{x}, \gamma, \alpha) \propto p(\boldsymbol{x} \mid \boldsymbol{\theta}) p(\lambda)
$$

where $p(\lambda)$ has been defined in (2). As a consequence

$$
\begin{aligned}
p(\lambda \mid \boldsymbol{x}, \gamma, \alpha) \propto & {\left[\prod_{i=1}^{n} p\left(x_{i} \mid \boldsymbol{\theta}\right)\right] p(\lambda) } \\
\propto & \frac{\delta^{n / \lambda}}{\gamma^{n / \lambda} \Gamma^{n}(1+1 / \lambda)} \mathbb{I}_{[0,3]}(\lambda) \\
& \times \exp \left[-\frac{\delta\left\|\boldsymbol{x}^{-}\right\|_{\lambda}^{\lambda}}{\gamma \alpha^{\lambda}}-\frac{\delta\left\|\boldsymbol{x}^{+}\right\|_{\lambda}^{\lambda}}{\gamma(1-\alpha)^{\lambda}}\right]
\end{aligned}
$$

where $\boldsymbol{x}^{+}$and $\boldsymbol{x}^{-}$contain all the positive and negative samples $x_{i}$ and $\|x\|_{\lambda}$ is the $l_{\lambda}$-norm. Unfortunately, this conditional distribution is not easy to sample directly. Thus, a random walk Metropolis Hastings (MH) move is used [7]. This move requires to define an appropriate proposal, which has been chosen as a zero mean Gaussian distribution whose variance has been adjusted a priori to obtain a suitable average acceptance ratio $r$. In practice, a reasonable range of $r$ is $30 \%$ to $90 \%$ and it is calculated within a sliding window of 30 samples. Note that to make sure the Markov chain is homogeneous after the burn-in period, this tuning procedure is only executed in the burn-in period (see [8] for more details).

The classical MH acceptance ratio necessary to ensure that the generated samples are asymptotically distributed according to their conditional distributions is

$$
\rho_{t}=\min \left\{\frac{p\left(\lambda^{*} \mid \boldsymbol{x}, \gamma, \alpha\right)}{p\left(\lambda^{t} \mid \boldsymbol{x}, \gamma, \alpha\right)}, 1\right\}
$$

where $\lambda^{*}$ is the candidate generated for iteration $t+1$ and $\lambda^{t}$ is the value of $\lambda$ at iteration $t$. 


\subsection{Sampling the scale parameter $\gamma$}

The conditional distribution of the scale parameter $\gamma$ is

$$
\begin{aligned}
p(\gamma \mid \boldsymbol{x}, \lambda, \alpha) & \propto p(\boldsymbol{x} \mid \boldsymbol{\theta}) p(\gamma) \\
& \propto\left[\prod_{i=1}^{n} p\left(x_{i} \mid \boldsymbol{\theta}\right)\right] \frac{1}{\gamma} \mathbb{I}_{\mathbb{R}^{+}}(\gamma) \\
& \propto \frac{1}{\gamma^{\frac{n}{\lambda}+1}} \exp \left[-\frac{1}{\gamma}\left(\frac{\delta\left\|x^{-}\right\|_{\lambda}^{\lambda}}{\alpha^{\lambda}}+\frac{\delta\left\|x^{+}\right\|_{\lambda}^{\lambda}}{(1-\alpha)^{\lambda}}\right)\right] \\
& =\mathcal{I} \mathcal{G}\left(\frac{n}{\lambda}, \frac{\delta\left\|\boldsymbol{x}^{-}\right\|_{\lambda}^{\lambda}}{\alpha^{\lambda}}+\frac{\delta\left\|\boldsymbol{x}^{+}\right\|_{\lambda}^{\lambda}}{(1-\alpha)^{\lambda}}\right)
\end{aligned}
$$

where $\mathcal{I} \mathcal{G}(a, b)$ is the inverse gamma distribution with hyperparameters $a$ and $b$.

Note that this distribution is easy to sample. This easy generation is mainly due to the form of APD introduced in (1), which differs from the definition of $[2,4]$.

\subsection{Sampling the asymmetry parameter $\alpha$}

The conditional distribution of the asymmetric parameter $\alpha$ satisfies the following relation

$$
p(\alpha \mid \boldsymbol{x}, \lambda, \gamma) \propto p(\boldsymbol{x} \mid \boldsymbol{\theta}) p(\alpha) .
$$

Using the definition of $p(\alpha)$ given in (4), the conditional distribution can be written

$$
\begin{aligned}
p(\alpha \mid \boldsymbol{x}, \lambda, \gamma) \propto & {\left[\prod_{i=1}^{n} p\left(x_{i} \mid \boldsymbol{\theta}\right)\right] p(\alpha) } \\
\propto & \frac{\delta^{n / \lambda}}{\gamma^{n / \lambda} \Gamma^{n}(1+1 / \lambda)} \mathbb{I}_{(0,1)}(\alpha) \\
& \times \exp \left[-\frac{\delta\left\|\boldsymbol{x}^{-}\right\|_{\lambda}^{\lambda}}{\gamma \alpha^{\lambda}}-\frac{\delta\left\|\boldsymbol{x}^{+}\right\|_{\lambda}^{\lambda}}{\gamma(1-\alpha)^{\lambda}}\right]
\end{aligned}
$$

Since this conditional distribution is not easy to sample directly, we have used an $\mathrm{MH}$ acceptance rule based on a uniform proposal in the interval $(0,1)$. Again, the simple form of APD introduced in (1) makes this generation easier since $\alpha \in(0,1)$ contrary to the APDs defined in [4] where $\alpha \in \mathbb{R}^{+}$.

\subsection{Minimum mean-squared error estimation}

The Bayesian estimators of the unknown parameters can be computed using the samples generated by the proposed hybrid Gibbs sampler. More precisely, the generated samples are averaged after an appropriate burn-in period to compute the minimum mean-squared error (MMSE) estimators of $\lambda, \gamma$ and $\alpha$.

\subsection{Numerical simulations}

Some numerical simulations have been run to evaluate the proposed estimation method. The first experiment considers
APD variates with parameters $(\lambda, \gamma, \alpha)$ that can be generated following the method proposed in [1], which is slightly modified to account for the scale parameter.

Fig. 1 shows an histogram of the generated data $x_{1}, \ldots, x_{n}$ (blue histogram) corresponding to the parameters $\alpha=0.75$, $\lambda=1.3$ and $\gamma=10$. The distributions of the samples generated by the proposed estimation algorithm are displayed in Fig. 2. The corresponding MMSE estimates \pm standard deviation, computed from 50 Monte Carlo runs, are $\hat{\alpha}=$ $0.748 \pm 0.03, \hat{\lambda}=1.297 \pm 0.022$ and $\hat{\gamma}=10.148 \pm 0.628$. These results are in good agreement with the true parameter values and confirm the good properties of the proposed Gibbs sampler. Finally, Fig. 1 also shows the good fit between the histogram of the generated data (in blue) and the APD pdf with the estimated parameters (in red).

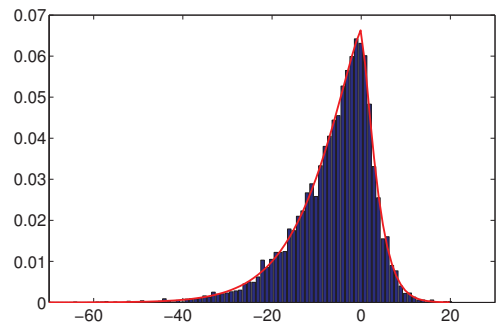

Fig. 1. Histogram of the generated APD random data (blue bars) and the corresponding estimated APD pdf (red line).
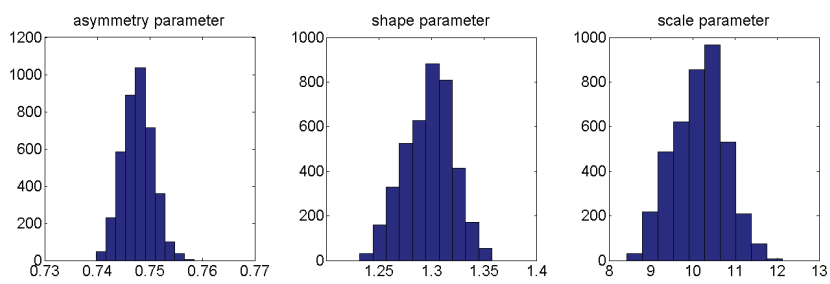

Fig. 2. Estimated marginal pdfs of the APD parameters.

\section{APPLICATION TO IMAGE SEGMENTATION}

This section shows that the algorithm developed before (to estimate the APD parameters) can be modified for an image segmentation application. More precisely, we assume that the image to be segmented is composed of homogeneous regions defined by different sets of APD parameters $(\alpha, \lambda, \gamma)$. The first part of this section describes the different steps of the proposed segmentation algorithm.

\subsection{Problem formulation}

Assuming the image is made up by $K$ homogeneous regions, a label vector $z \in\{1, \ldots, K\}^{n}$ mapping each image pixel into the set $\{1, \ldots, K\}$ is defined. The distribution of the pixel $x_{i}$ 
conditionally on the $k$ th class is supposed to be defined as

$$
x_{i} \mid z_{i}=k \sim A P D\left(\boldsymbol{\theta}_{k}\right)
$$

where the APD parameter vector $\boldsymbol{\theta}=(\lambda, \gamma, \alpha)^{T}$ is associated with the $k$ th class. Assuming the pixels are independent conditionally to the knowledge of their classes, we obtain the following prior for the target image

$$
p(\boldsymbol{x} \mid \boldsymbol{z}, \boldsymbol{\theta})=\prod_{k=1}^{K} \prod_{i=1}^{n_{k}} f_{A P D}\left(x_{i} \mid z_{i}=k, \boldsymbol{\theta}_{k}\right)
$$

where $n_{k}$ is the number of pixels in class $\# k$ and $\boldsymbol{\theta}=$ $\left(\boldsymbol{\theta}_{1}^{T}, \ldots, \boldsymbol{\theta}_{K}^{T}\right)^{T}$.

The prior of the image labels is supposed to be a Markov random field (MRF) to take advantage of the dependencies between neighbor pixels in the image. The conditional distribution of $z_{i}$ for an MRF is defined as

$$
p\left(z_{i} \mid \boldsymbol{z}_{-i}\right)=p\left(z_{i} \mid \boldsymbol{z}_{\nu(i)}\right)
$$

where $\boldsymbol{z}_{-i}=\left(z_{1}, \ldots, z_{i-1}, z_{i+1}, \ldots, z_{i}\right)$ and $\nu(i)$ contains the neighbors of label $z_{i}$. The whole set of random variables $z_{i}$ forms a random field. The Potts Markov field defined by the neighborhood structure (14) is particularly adapted to labelbased segmentation [9]. Using the Hammersley-Clifford theorem [10], the prior of $z$ can be expressed as a Gibbs distribution

$$
p(\boldsymbol{z})=\frac{1}{C(\beta)} \exp \left[\Phi_{\beta}(\boldsymbol{z})\right]
$$

with

$$
\Phi_{\beta}(\boldsymbol{z})=\sum_{i=1}^{n} \sum_{i^{\prime} \in \nu(i)} \beta \delta\left(z_{i}-z_{i^{\prime}}\right)
$$

where $\beta$ is the granularity coefficient, $\delta($.$) is the Kronecker$ function and $C(\beta)$ is the normalizing constant. In this paper the value of $\beta$ has been fixed to 1.2 by cross validation.

The image segmentation problem addressed in this paper consists of estimating the label vector $z$ and the parameter vectors $\boldsymbol{\theta}_{k}$ for $k=1, \ldots, K$ from the image $\boldsymbol{x}$. We propose to study Bayesian estimators of $(\boldsymbol{\theta}, \boldsymbol{z})$ based on the following posterior distribution

$$
p(\boldsymbol{z}, \boldsymbol{\theta} \mid \boldsymbol{x}) \propto p(\boldsymbol{x} \mid \boldsymbol{z}, \boldsymbol{\theta}) p(\boldsymbol{z}) p(\boldsymbol{\theta})
$$

with

$$
p(\boldsymbol{\theta})=\prod_{k=1}^{K} p\left(\boldsymbol{\theta}_{k}\right)=\prod_{k=1}^{K}\left[p\left(\lambda_{k}\right) p\left(\gamma_{k}\right) p\left(\alpha_{k}\right)\right]
$$

The posterior (17) is too complex to derive closed form expressions of the Bayesian estimators of $(\boldsymbol{\theta}, \boldsymbol{z})$. As a consequence, we propose to sample $p(\boldsymbol{z}, \boldsymbol{\theta} \mid \boldsymbol{x})$ by using a hybrid Gibbs sampler presented in the next section.

\subsection{Hybrid Gibbs sampler}

After an appropriate initialization, the proposed hybrid Gibbs sampler is made of 4 steps

1. Sampling the shape parameter vector $\boldsymbol{\lambda}=\left(\lambda_{1}, \ldots, \lambda_{K}\right)^{T}$

2. Sampling the scale parameters $\gamma=\left(\gamma_{1}, \ldots, \gamma_{K}\right)^{T}$

3. Sampling the asymmetry parameters $\boldsymbol{\alpha}=\left(\alpha_{1}, \ldots, \alpha_{K}\right)^{T}$

4. Sampling the labels $z$

The first three sampling steps are very similar to those presented in Section 4. The only modification appears in the expression of the conditional distributions which depend on the different labels. For example, the conditional distribution of the shape parameter $\lambda_{k}$ can be written

$$
p\left(\lambda_{k} \mid \boldsymbol{x}_{k}, \boldsymbol{z}_{k}, \alpha_{k}, \gamma_{k}\right) \propto p\left(\boldsymbol{x}_{k} \mid \boldsymbol{z}_{k}, \boldsymbol{\theta}_{k}\right) p\left(\lambda_{k}\right)
$$

with

$$
p\left(\boldsymbol{x}_{k} \mid \boldsymbol{z}_{k}, \boldsymbol{\theta}_{k}\right)=\prod_{i=1}^{n_{k}} p\left(x_{i} \mid z_{i}=k, \boldsymbol{\theta}_{k}\right) .
$$

The last sampling step is required to define the conditional distribution of the label vector $\boldsymbol{z}$. Following [8], the conditional distribution of the labels $z$ can be computed using the Bayes rule

$$
p(\boldsymbol{z} \mid \boldsymbol{x}, \boldsymbol{\theta}) \propto p(\boldsymbol{x} \mid \boldsymbol{z}, \boldsymbol{\theta}) p(\boldsymbol{z}) .
$$

Considering the dependency between a label and its neighbors, the conditional distribution of the label $z_{i}$ (corresponding to image pixel $x_{i}$ ) is given as follows

$$
p\left(z_{i}=k \mid \boldsymbol{z}_{-i}, \boldsymbol{x}, \boldsymbol{\theta}\right) \propto p\left(x_{i} \mid z_{i}=k, \boldsymbol{\theta}\right) p\left(z_{i}=k \mid \boldsymbol{z}_{\nu(i)}\right) .
$$

Denoting as $\pi_{i, k}=p\left(\boldsymbol{z}_{i}=k \mid \boldsymbol{z}_{-i}, x, \boldsymbol{\theta}\right)$, the normalized conditional probability of the label $z_{i}$ is

$$
\tilde{\pi}_{i, k}=\frac{\pi_{i, k}}{\sum_{k=1}^{K} \pi_{i, k}} .
$$

The label $z_{i}$ can be drawn from the set $\{1, \ldots, K\}$ with the respective probabilities $\left\{\tilde{\pi}_{i, 1}, \ldots, \tilde{\pi}_{i, K}\right\}$. Note finally that a fourpixel neighborhood structure has been adopted in this paper.

\subsection{Experiments}

The proposed algorithm has been applied to a synthetic image composed of four areas defined by APDs with different parameters reported in Table 1 . Each area contains $100 \times 100$ pixels yielding an image of $200 \times 200$ pixels. An example of a generated image using the considered APD parameters is displayed in Fig. 3 (a). The corresponding segmentation map obtained with the proposed algorithm is shown in Fig. 3 (b). It is clearly in good agreement with the ground truth (the rate of correct segmentation is equal to $99.7 \%$ ).

Table 2 provides more quantitative results in term of means and standard deviations for the estimated APD parameters computed from 50 Monte Carlo runs with the same parameter values. From these results, after comparing the true and 


\begin{tabular}{|c|c|c|c|c|}
\hline & Area 1 & Area 2 & Area 3 & Area 4 \\
\hline$\alpha$ & 0.75 & 0.35 & 0.5 & 0.15 \\
\hline$\lambda$ & 1.5 & 2 & 1.7 & 1.2 \\
\hline$\gamma$ & 10 & 2 & 5 & 15 \\
\hline
\end{tabular}

Table 1. APD parameters of the areas of the synthetic image.

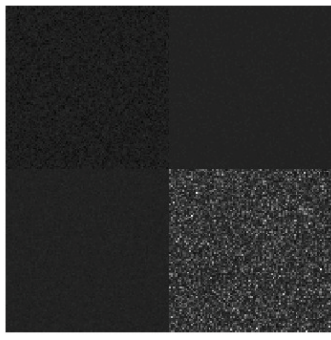

(a)

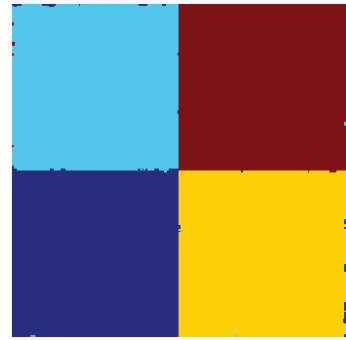

(b)
Fig. 3. Representative example of (a) a synthetic image made of four areas with different APD parameters (as indicated in Table 1) and (b) a segmentation result.

the estimated parameter values, one can wonder whether the estimation is correct and effective, especially for the largest values of $\gamma$. To illustrate this accuracy, Fig. 4 compares the histograms of the generated data for each area (blue bars) with the theoretical marginal posterior distributions (in red) and the estimated marginal posterior distribution (in green). Even if there are some differences between the estimated and the true parameter values, the green curves are superimposed with the red curves showing that the estimated APD parameters provide a very good data fit.

\begin{tabular}{|c|c|c|c|c|}
\hline & Area 1 & Area 2 & Area 3 & Area 4 \\
\hline$\hat{\alpha}$ & $0.756 \pm 0.003$ & $0.346 \pm 0.003$ & $0.498 \pm 0.003$ & $0.138 \pm 0.002$ \\
\hline$\hat{\lambda}$ & $1.613 \pm 0.035$ & $2.128 \pm 0.054$ & $1.843 \pm 0.043$ & $1.268 \pm 0.024$ \\
\hline$\hat{\gamma}$ & $12.580 \pm 0.956$ & $2.075 \pm 0.089$ & $6.101 \pm 0.417$ & $17.254 \pm 1.343$ \\
\hline
\end{tabular}

Table 2. Estimated APD parameters for each image area.

\section{CONCLUSIONS}

This contribution proposed a new Bayesian model for estimating the parameters of an asymmetric power distribution. A Gibbs sampler allowing the parameters of this model to be generated was also studied. An application to image segmentation was finally investigated. The obtained simulation results showed the efficiency of the proposed estimation method approach for both parameter estimation and image segmentation. Future work will be devoted to the analysis of real images with the proposed Bayesian framework.

\section{REFERENCES}

[1] I. Komunjer, "Asymmetric power distribution: Theory and applications to risk measurement," Journal of applied econometrics, vol. 22, pp. 891-921, 2007.
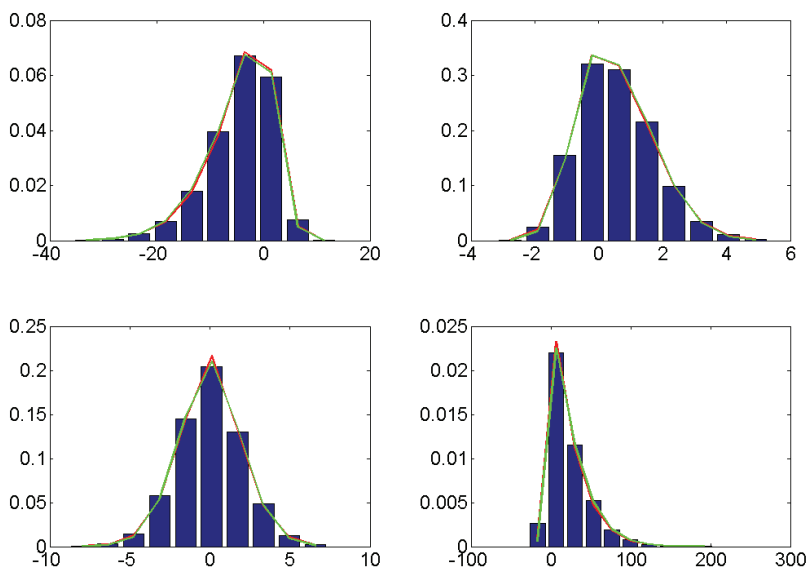

Fig. 4. Marginal posterior distribution from the estimated (green) and true (red) APD parameters in each area of the image in Fig. 3.

[2] D. Zhu and V. Zinde-Walsh, "Properties and estimation of asymmetric exponential power distribution," Journal of econometrics, vol. 148, pp. 86-99, 2009.

[3] N. Nacereddine, S. Tabbone, D. Ziou, and L. Hamami, "Asymmetric generalized gaussian mixture models and EM algorithm for image segmentation," in International Conference on Pattern Recognition, Istanbul, Turkey, 2010.

[4] N-E. Lasmar, A. Baussard, and G. Le Chenadec, "Asymmetric power distribution model of wavelet subbands for texture classification," Pattern Recognition Letters, vol. 52, pp. 1-8, 2015.

[5] L. Chaâri, J-C. Pesquet, J-Y. Tourneret, Ciuciu P., and A. Benazza-Benyahia, "A hierarchical Bayesian model for frame representation," IEEE Trans. Signal Processing, vol. 58, no. 11, pp. 5560-5571, 2010.

[6] C. P. Robert and G. Casella, Monte Carlo Statistical Methods, Springer Texts in Statistics, 2004.

[7] W. K. Hastings, "Monte Carlo sampling methods using Markov chains and their applications," Biometrika, vol. 57, no. 1, pp. 97-109, 1970.

[8] N. Zhao, A. Basarab, D. Kouame, and J-Y. Tourneret, "Joint segmentation and deconvolution of ultrasound images using a hierarchical Bayesian model based on generalized Gaussian priors," submitted to IEEE Trans. Image Processing, 2015.

[9] M. Pereyra, N. Dobigeon, H. Batatia, and J-Y. Tourneret, "Estimating the granularity coefficient of a Potts-Markov random field within a Markov chain Monte Carlo algorithm," IEEE Trans. Image Processing, vol. 22, no. 6, pp. 2385-2397, 2013.

[10] J. Besag, "Spatial interaction and the statistical analysis of lattice systems," Journal of the Royal Statistical Society. Series B, vol. 36, no. 2, pp. 192-225, 1974. 